\title{
Theoretical Models for Sport Participation: Literature Review
}

\author{
Simon Grima ${ }^{1}$, Alan Grima ${ }^{2}$, Eleftherios Thalassinos ${ }^{3}$, Sharon Seychell ${ }^{4}$, Jonathan \\ V. Spiteri ${ }^{5}$
}

\begin{abstract}
:
Recent studies have analyzed theoretical models of sport participation. They claimed that sports activities relate to health and happiness and that there are various factors which determine sports participation, be it individual, sociological or psychological. Whilst some countries in Europe experienced an increase in sport activity over the past few decades, others saw a decline in the number of individuals who commit to physical activity. Several models have been constructed to explain and determine involvement in sports namely, 'The Beckerian Approach', 'The SLOTH framework' and 'Green's Model of Sport Development'. These models have unearthed specific important factors, which encourage people to take part in sports activities. These concerned age, gender, time constraints, income and level of education.
\end{abstract}

In fact, findings has shown a positive and statistically significant relationship between age and frequency of sport participation and as age increased walking increased as well. Education plays an important role as well especially where adolescents are concerned. School was a key contributor to adolescents increased participation in physical activity and more specifically as they transitioned into secondary school.

A successful example is that of Norway where a sport and physical recreation culture is deeply rooted in society and is supported by strategic socio-economic circumstances, high standards of living, equality between genders, abundant sporting facilities, a school system that keenly promotes physical activity, a strong voluntary sports clubs sector and high levels of parental contribution. Recent research has also focused on the sociological and psychological factors which contribute to increased physical activity. Social networks and friends significantly impact one's decision to take part in sport, while the involvement of parents in sport affects sport frequency in a positive and significant way.

Keywords: Sport participation, multi-level analysis.

\footnotetext{
${ }^{1}$ University of Malta, Head Insurance Department, Faculty of Economics, Management and Accountancy corresponding author, simon.grima@um.edu.mt

${ }^{2}$ University of Malta, Faculty of Economics, Management and Accountancy, alan_grima4@hotmail.com

${ }^{3}$ University of Piraeus, Faculty of Industrial and Maritime Studies, thalassinos@ersj.eu

${ }^{4}$ University of Malta, Insurance Department, Faculty of Economics, Management and

Accountancy, sharonseychell@gmail.com

${ }^{5}$ University of Malta, Insurance Department, Faculty of Economics, Management and

Accountancy, jonathan.v.spiteri@um.edu.mt
} 


\section{Introduction}

The second half of the last century witnessed a significant increase in numbers and frequency of sport participation in Europe (Gratton and Taylor, 2000). However, recently, some European countries such as Italy and the Netherlands (Van Bottenburg, 2005) have experienced a decline in sport participation. The participation level in other countries, like for instance Austria, Finland, Portugal (Van Bottenburg, 2005), England (Sport England, 2010) and Spain (Garcia and Llopis, 2011) have remained stagnant. This negative development in sport participation and the increased health-related concerns mushrooming across several continents have fueled a strong academic interest in this field of research.

Traditionally, a considerable amount of literature on sport had an economic, demographic and sociological background and focused primarily on the several impacts of sports and physical activity on the individual, community and population. Nowadays, there is growing evidence available on the determinants of sport participation and physical activity and the impacts these have on health and labour market outcomes as well as other essential societal objectives. One of several government policy objectives in many countries aims to ameliorate participation in sport and other physical activity at all levels of the population.

Several approaches have been used to analyze sport and physical activities and hence a perfect evaluation of their determinants may be difficult. Downward et al., (2011) provide four caveats to explain that a certain amount of caution must be exhibited when reviewing sport participation evidence:

- The list of sporting activities varies between studies;

- Different ways are employed to measure the sport participation variable;

- The use of different statistical approaches makes comparability of estimates difficult in both sign and magnitude;

- The determinants of sport participation are peculiar to different countries.

Notwithstanding the above, it is still possible to make general assessments on the different variables of sport participation.

\section{Literature Review}

The Beckerian Approach (1965, 1974): Sport participation has been effectively explained through the "Income-Leisure Trade-Off Model of Labour Supply" (Downward and Riordan, 2007; Hallmann et al., 2011; Ruseski et al., 2011; Wicker et al., 2009). This model uses the household as its unit of analysis (Becker, 1965). The household production theory refers to several economic choices. Individuals produce and consume basic commodities such as going to the cinema or out for dinner by allocating resources for instance, time and income to them. The relative intensity of these contributions is fundamentally important for such choices. Sports 
participation falls into the same category. According to Becker (1974), individuals also make decisions to invest into personal capital and skills through the allocation of time and other goods. The demand for sport can also be influenced by other persons' characteristics.

The SLOTH framework developed by Cawley (2004): This is based on Becker's (1965) model of labour and leisure choice and was used by a number of researchers aimed to investigate economic decisions influencing participation in physical activity. This model "incorporates the idea that individuals produce their own health" (Eberth and Smith, 2010). In this context, Cawley "assumes that utility depends on an individual person's weight, health, food, and other goods and time spent" (Garcia et al., 2011) based on SLOTH which is an acronym for sleeping, leisure, occupation, transportation and home production. Humphreys and Ruseski (2006; 2007) developed an extended version of SLOTH to analyze physical activity in the United States.

Green's (2005) Model of Sport Development: established a sport development model to examine factors related to sport participation. This model comprises three stages called recruitment, retention and transition. It aims to comprehend the factors which are critical to the development of sport, more precisely the combination of factors which impact participation rates and the commitment to sport of individuals. In every stage of this model, several motivations and available opportunities exist which directly influence an individual's decision to carry on or exit sport.

Recruitment is the first stage in the sport development model process which involves the individual, family and sport delivery system levels that influence an individual's decision to take part in sport. Motivations play an important role at the individual level which in addition to the availability of sports and sport programs, indicative of the system level, increase the likelihood that individuals move into a sport that better meets their needs and motivations.

Retention is a very challenging, but many times ignored stage in the process. Retaining and possibly increasing the involvement of individuals requires an indepth analysis of what is that affects their decision to stay. Financial resources, skill level and social support are constraints that can affect the commitment of individuals towards a sport. Lack of sport programmes or coaching availability at the system level can disturb their commitment.

Various transitions occur between the entrance and retention stages. These take place either in life stages (example: single to married or youth to adult) and commitment stages (example: recreational to elite). Similarly, to the other two stages, transitions are also marked by individual differences, motivations and delivery system factors. Here, utmost attention must be given to certain aspects which encourage further participation such as providing more training opportunities, encouragement to advance and flexibility in membership options. 
A Sport Participation Model based on Becker: The economical time-allocation (economic behaviour) theory of Becker (1965) prepared the ground to a general theoretical model of sports participation. The decision to participate in sports is also based on monetary and time restrictions. This theory was further developed to include several demographic and social variables and has been usefully used in previous research. Breuer (2006), Downward (2007), Downward and Riordan (2007), Hallmann et al. (2011), Hallmann and Breuer (2014) and Wicker et al. (2009) have applied the micro-level measure of the model in their research on sports participation.

A Multi-Level Model of Sport Participation: The model consists of two sides or two levels - factors on the individual level (demand side) and factors on the infrastructure level (supply side). Other research refers to these two levels as the micro and macro levels. Wicker et al. (2012) also incorporated the macro-level factors in the form of sport facilities and sport programmes transforming it into a multi-level analysis approach. Many times, research analyzed the availability of sports facilities by identifying their quantity and location.

\section{Determinants of Sports Participation}

Individual or Micro-Level Factors (Sport Demand): Various studies define microlevel factors as the individual factors which directly relate to the individual person. Wicker et al. (2012) Hallmann et al. (2011) Downward et al. (2009) provide a detailed description of the micro-level factors. They also observed several tendencies when analyzing the effect of these factors on sports participation. Microlevel factors can be divided into two sections: demand-specific factors namely age, gender and migration background and household-economic factors, namely human capital, income and time.

The Demand-Specific Factors - Demographic Variables: Sports participation may also be influenced by the demand-specific factors or more precisely the demographic variables (Breuer et al., 2010; Downward and Riordan, 2007; Hallmann et al., 2011). Differences in behaviour are attributed to gender. Empirical evidence reveals that men are significantly more active than women indicating gender can be a barrier for sports participation (Berger et al., 2008; Breuer et al., 2011; Breuer and Wicker, 2008; Downward, 2007; Downward and Rasciute, 2011; Eberth and Smith, 2010; Haug, et al., 2008; Humphreys and Ruseski, 2006; Robertson and Emerson, 2010; Seabra et al., 2007; Federico et al., 2013; Hovemann and Wicker, 2009; Taks and Scheerder, 2006; Van Tuyckom et al., 2010).

Different social, cultural and biological factors affect decisions of men and women to take part in sports and the relative frequency of such participation. Women experience greater difficulties to access sport due to provision of facilities, commuting and time obligations (Downward et al., 2014). In fact, the authors suggest that more childcare provision could help achieve more female participation. 
Certain social or cultural influences and differences in family responsibilities are congruent with realities countries (Humphreys and Ruseski, 2011). Having children and housekeeping are two main restrictions (Downward, 2007; Eberth and Smith, 2010; Hovemann and Wicker, 2009; Wicker, et al., 2009). However, families could also reduce the intensity of male participation (Downward and Rasciute, 2015). There was no significant effect of age on sports participation in Wicker et al., (2012).

A qualitative study on working mothers by Dixon (2009) suggests that women spend less time in leisure and sport activities than men, while the latter take part more often and in more sports at all stages of life, implicating clearly gender inequity in this regard. Henderson and Hickerson (2007) also identified lack of knowledge, interest and opportunity as critical barriers for women. Lim et al., (2011) argued that women are impacted by their capability to comprehend rules and norms. Higher education and income are synonymous of more physically active Brazilian women aged between 20 and 40 years (Balbinotto et al., 2012). A recent study reveals that women tend to adopt less intensive activity even if they have time at their disposal (Downward and Rasciute, 2015).

During the adolescence period of life, girls reported more statistically significant decreases in physical activity than boys (Lubans et al., 2007; Zimmermann-Sloutkis et al., 2010). In a study conducted in South Australia, Slater and Tiggemann (2010) identified several genders-specific reasons hindering adolescent girls from physical activity. Reasons varied from insufficient time, lack of competence, apprehensions about physical appearance, reduced interest and boredom, to relationships with teammates and teasing, and incompatibility of certain sport with femininity.

Eime et al. (2013) concluded that school was a key contributor to adolescents increased participation in physical activity significantly and more specifically as they transitioned into secondary school. However, adolescents also significantly reduce participation between year 10 and year 11 in addition to shifting towards a more non-organized and non-competitive forms and individual pursuits of physical activity. Eime et al. (2015) have "identified significant and interrelated patterns of change in intrapersonal, interpersonal and environmental determinants of participation in PA by girls across the adolescent period". Intrapersonal limitations linked to perceived competence and lack of energy and time; interpersonal factors include support by family and friends whereas opportunity, resources and access were associated with environmental issues.

However, in contrast, a study investigating sports participation in EU revealed that Danish and Netherlands women are significantly more active than men (Hovemann and Wicker, 2009). Likewise, women were found to be more frequently active than men (Humphreys and Ruseski, 2006, 2007; Lera-López and Rapún-Gárate, 2011). 
Breuer and Wicker (2009) reveal that contrasting results can be attained depending on the method of analysis employed. Declines in participation levels with increasing age are supported by cross-sectional analysis while longitudinal analyses imply that cohort effects not age are responsible for such declines. In their study, all women cohorts except the oldest established increasing sport participation rates.

In addition, Lim et al. (2011) revealed that both males and females' sports participation in the Netherlands, Republic of Korea and USA declines as they move into adult life. Hence, the analysis by gender, similarly to the other demand-specific factors plays a vital role in the theoretical multi-level model of sport participation.

Previous empirical evidence has shown that the younger generation tends to be more active than the senior one, and hence sports participation decreases as age increases (Berger et al., 2008; Breuer and Wicker, 2008; Chad et al., 2005; Downward, 2007; Eberth and Smith, 2010; Fridberg, 2010; Humphreys and Ruseski, 2009; Van Tuyckom and Scheerder, 2008). One main reason for this restriction on sport demand is that more health problems connected with biological and physical limitations are experienced as a person grows older, thus effecting sports participation or the time spent in it negatively (Downward et al., 2011; Downward, 2007; Eberth and Smith, 2010; Humphreys and Ruseski, 2011). Changes in the type of sport practiced as age increases have also been noted. In fact, walking is positively associated with age (Humphreys and Ruseski, 2007; Lera-Lopez and Rapun-Garate, 2011).

Klein (2009) states that increasing age up to 50 years is associated with increasing physical activity. On a different note, Garcia et al., (2011) discovered that "as age increases, the probability of doing sports decreases up to the age of 33, after which the relationship is reversed". Moreover, elderly women participate less in sporting activities than elderly men (Hinrichs et al., 2010).

Lera-López and Rapún-Gárate (2011) revealed a positive and statistically significant relationship between age and frequency of sport participation. Hence, frequency of sport participation increases as age increases, revealing older people's increased awareness of the already described benefits and willingness to remain active in sustainable forms of physical activity. After all, older people have more time for such healthy engagements. Hallmann and Breuer (2014) also found that older people take part in sports. Still, the probability for individuals to take part in sport falls $0.3 \%$ for every additional year of age (Humphreys and Ruseski, 2006).

Special care must be displayed in view of the migration background as literature indicates use of different terms and meanings. Any comparisons require a certain degree of caution. Migration background and nationality are under study in Europe (Snape and Binks, 2008; Walseth, 2008) while ethnicity is examined in North America and the UK (Stamatakis and Chaudhury, 2008). These studies have exposed evidence related to this demand-specific factor. People with a migration background 
tend to experience cultural barriers and so are likely to be less active than those without a migration background (Snape and Binks, 2008). Sports participation is also high for white ethnic people (Stamatakis and Chaudhury, 2008). Wicker et al. (2012) reveal a negative impact of the migration background factor on "all sport participation and sport activity in non-profit sport clubs".

Consequently, with such findings, one can possibly assume that culture, national traditions and values not only play a vital role on sports participation within a country but also when comparisons are made between different countries. In this regard, Van Tuyckom and Scheerder (2010) have revealed a very interesting fact which indirectly concerns Malta. They found that inhabitants in Southern Europe and Mediterranean Sea countries are less active than their Northern counterparts including Scandinavian countries.

The Household-Economic Factors - Socio-Economic Variables: Studies by Chad et al. (2005), Eberth and Smith (2010) and Humphreys and Ruseski (2006) indicate that individuals with a higher level of human capital or educational background are more conscious about the positive outcomes of sports and therefore are more likely to participate in it. The positive impact of a good educational background on sport participation is also supported by other studies in specific European countries (Breuer et al., 2011; Breuer and Wicker, 2008; Downward and Rasciute, 2011; Fridberg, 2010; Hovemann and Wicker, 2009; Humphreys and Ruseski, 2007; Ifedi, 2008; Scheerder et al., 2006; Scheerder and Vos, 2011; Wicker et al., 2009).

The efficiency of an individual's household production is affected by human capital. A higher educational level is understandably linked with higher income and more sports participation (Hallmann et al., 2011). Downward and Rasciute (2015) point out that higher education levels play a bigger role for females. Hallmann and Breuer (2014) reveal thought-provoking insights on the possible influences of education on sports frequency. Their results indicate a significant negative influence most probably due to the increased time restrictions placed on individuals in higher levels of education.

Another important economic factor is income. Evidence through empirical studies indicates that higher income supports sports participation (Breuer and Wicker, 2008; Breuer et al., 2011; Downward and Rasciute, 2010; Eberth and Smith, 2010; Humphreys and Ruseski, 2007; Lera-López and Rapún-Gárate, 2007; Scheerder and Vos, 2011; Stamm and Lamprecht, 2011; Wicker et al., 2009). Low income limits sport participation for men and women alike (Eberth and Smith, 2010). Lera-López and Rapún-Gárate (2011) insist that once individuals commit participation than such factor is no more significant in defining frequency levels. The same study classifies occupations or professional status such as self-employed, manager, clerical worker, unemployed and entrepreneur as negative determinants of sport participation. 
On the other hand, sports participation frequency is not influenced (Gratton and Taylor, 2000) or negatively influenced (Downward and Riordan, 2007; Garcia et al., 2011; Humphreys and Ruseski, 2011) by the level of income of the individual. Some sporting activities can be quite expensive. Moderately to high expensive sports such as tennis are not practiced by people with lower income (Taks et al., 1994). Increased participation in sports is related with a higher level of income thus creating better access opportunities to sport (Humphreys and Ruseski, 2009; Berger et al., 2008; Ifedi, 2008; Downward, 2007).

It is also necessary to take into consideration the consumer behaviour in relation to sport expenditure since some sporting goods and services need to be consumed to take part in sport. Analysis of sport expenditure is not much extensive and there is lack of appropriate methodologies. Still, men seem more likely to spend more money on sports than women (Lera-López and Rapún-Gárate, 2005; 2007). Higher spending on sports is related to higher levels of education (Lera-López and RapúnGárate, 2005). Higher income plays a statistically significant influence on sport consumption (Lera-López and Rapún-Gárate, 2011). Garcia et al. (2011) showed that the relative demand for physical activity declines with higher hourly earnings because of the increased opportunity cost of time consumed on any leisure activity. Males enunciate this effect more.

Breuer et al. (2010) concluded that "consumer expenditure on sports is mainly determined by gender, education, and income level (spending decreases for females but increases with education and income)". Similarly, Thibaut et al. (2014) revealed that household spending behaviour was positively influenced by "family income, education of the head of the household, sports participation of the parents during their youth, sports club membership and the frequency of sports participation".

A third key factor is time. The time available for sports participation is influenced by two variables: occupation and household size. In other words, it is deduced after reducing the time for competing demands such as working time and time spent caring for children and relatives. Studies by Breuer (2006) and Downward (2007) confirm that time for sports participation increases as time for work and care decreases. Individuals with more time constraints are more likely to take part in less intensive or prolonged activities (Hallmann et al., 2011). Retired people are more likely to participate in sports than the employed (Eberth and Smith, 2010). Contrarily, findings by Hallmann and Breuer (2014), Wicker et al. (2009) and Wicker et al. (2012) state that working time had a positive impact on sport participation, with the most probable motivation related to a compensation effect placed on sport in return for high working loads.

Evidence exposed the role played by the household profile. Downward (2007) and Humphreys and Ruseski (2007) revealed that the household size is negatively associated with sport participation. Sport participation of females is decreased by 
the presence of more adults and children in the household (Downward et al., 2014; Eberth and Smith, 2010). Married couples have less time for sports participation and alternate physical activities (Humphreys and Ruseski, 2006). The time spent caring for children and relatives was found to pose a negative influence on sport participation (Downward, 2007; Eberth and Smith, 2010; Hovemann and Wicker, 2009; Humphreys and Ruseski, 2006; Klein, 2009; Wicker et al., 2009) except for Wicker et al. (2012) who found no significant influence.

Work, household, sport and leisure commitments force individuals to face daily conflicting decisions regarding the allocation of time and income which are a fundamental part of this framework. Employment can have a negative impact on sports participation, possibly attributable to time substitution (Breuer and Wicker, 2008; Downward, 2007; Eberth and Smith, 2010; Hovemann and Wicker, 2009; Lera-López and Rapún-Gárate, 2011). Many times, several trade-offs take place when individuals attempt to assign time and money to the various activities they engage in on a daily basis. The days of the week and the time of the year also play their part. Weekends and spring and summer seasons increase the probability of playing sports (Garcia et al., 2011).

A study by Anoyke et al. (2014) explored the association of time and money with physical activity using a nationwide dataset in England. It revealed an association between lower participation in physical activity and high travel time and money prices per occasion of physical activity. The latter resulted from parking fees, facility charges, child care and family members' fees. The study suggested positive financial incentives measures such as subsidizing the price of participation to counteract this trend.

\section{Sports Infrastructure or Macro-Level Factors (Sport Supply)}

Adequate sport infrastructure is important to sport participation, as many sports cannot be performed without having the appropriate sport facility (Hallmann et al., 2012). We define sports infrastructure as the basic facilities, services and installations serving sports organizations, sports users and other community members providing increased opportunities to all to participate in sport for leisure, training or competitive purposes. Sport infrastructure includes sports facilities (sports halls, sport pitches, playing courts and swimming pools) and sport programmes (operated by sport clubs, commercial providers and city). Some studies also include park areas and similar recreational areas in their research (Humphreys and Ruseski, 2007; Wicker et al., 2012).

Macro-level factors play a positive role on sport activity and thus can be considered as facilitators of sports participation. A positive effect on sport activity was witnessed by a larger supply of sports facilities and sport programmes (Chad et al., 2005; Haug et al., 2008; Limstrand and Rehrer, 2008). A host of studies have further established that physical activity is positively correlated with the availability of 
suitable sport infrastructure (Chad et al., 2005; Haug et al, 2008; Liu et al., 2009; Wicker et al., 2012). However, Downward et al. (2011) found that "the availability of sports facilities is not statistically significant in explaining the decision to participate in sport activities". It is sometimes difficult to compare these results as each study operationalized sports supply in different ways, in the sense that it was taken from different perspectives and included differences in the type of infrastructures as well as sizes and locations of cities.

Sport infrastructure plays an important part in anticipating sports participation, even though this is dependent on the type of sport and facility (Wicker et al., 2012; Wicker et al., 2013). More sport participation is experienced when individuals get a feeling of satisfaction with the use of facilities (Downward and Rasciute, 2011). It has also been demonstrated that the frequency of sport activities is reduced if there are less sports facilities available. Wicker et al. (2009), Hallmann et al. (2011) and Haug et al. (2008) insist that an important barrier to sports participation is in fact insufficient infrastructure. It also transpires that sports clubs and federations have a very important role to play. Downward et al. (2011) reveal that the frequency of sport participation is highly influenced by sports clubs' memberships and federations.

Research about the use of sports facilities shows gender differences. Boys use soccer fields and ski/snowboarding resorts whereas girls opt for beaches, skating rinks and playgrounds (Haug et al., 2008). Likewise, Downward and Rasciute (2015) reveal that males use grass pitches to play team sports, while females enjoy swimming and keep fit activities. The latter study also indicates an imbalance in favour of male sports facilities, resulting in females deriving less satisfaction with sports facilities.

Proximity of sports facilities encourages the participation of more people (Limstrand and Reher, 2008). Downward et al. (2014) claim that males consider the provision of sport infrastructure more important than females. Contrarily, "improved proximity to gyms is likely to be more important for female adolescents living in rural areas" in Germany (Reimers et al., 2014). A negative impact on sports participation could arise in large cities where the availability of entertaining facilities is greater than sport infrastructure which consequently translates into a substitution effect (Garcia et al., 2011). To counter act this, Van Tuyckom and Scheerder (2010b) found that large towns are more physically active. The results of a study about Dutch adolescents, point out that "leisure time sports participation is associated with levels of neighborhood social capital, but not with availability of parks or sports facilities" (Prins et al., 2012).

In France, Karusisi et al., (2013) findings backed "evidence that strategies to increase participation in sport activities should improve the spatial and financial access to specific facilities, but also address educational disparities in sport practice". Eime et al., (2015) demonstrated that the association between the 
relationship, frequency and organisational context of participation with SocioEconomic-Status (SES) and location is quite complex. While it seems unfitting to generalize about to SES and location, only a few forms of physical activity were found to be cost- or remoteness- unreasonable in terms of participation. To this end, both SES and location stand as insignificant determinants of the depth of involvement in physical activity once this is established.

Physical activity and sports participation in Netherlands, Republic of Korea and USA were investigated in a qualitative study carried out by Lim et al. (2011). Their findings indicated that individual, household and neighborhood socio-economic status are all related to physical activity and sports participation. Evidence also suggests that higher SES neighborhoods reported significantly more physical activity facilities than lower SES neighborhoods, hence providing more opportunities to be physically active. The three countries also experienced other similar barriers to physical activity and sport participation mainly in the form of time pressure and costs. The authors of this study made use of some stages from Green's (2005) sport development model to analyze individual and structural factors that have an impact on adult levels and patterns of sport participation. He argues that sport has evolved in a variety of ways in different parts of the world and thus the sport systems and structures employed in a country impact its sport development and participation.

Stahl et al. (2001) claim that the physical environment, such as sports facilities, are the "best marker" for ensuring that people are physically active. Haug et al. (2008) found an association between the availability of outdoor facilities in secondary schools and students' participation in physical activity during break. Another study carried out in Stuttgart, Germany by Wicker et al (2009) and which used hierarchical linear models the general implication of sport infrastructure indicates that irrespective of individual socio-economic conditions the availability of sport infrastructure influences patterns of sport activity significantly. The data obtained also suggests that different age groups have different needs for sport and hence during life the type of sport infrastructure needed also changes.

In Norway, Limstrand and Rehrer (2008) also obtained similar results even though they were not able to confirm that physical activity rises because of the provision of sports facilities among all young people. The extensive growth of sports participation in China came because of a nationwide policy which saw the Chinese government investing heavily on sports infrastructure (Xiong, 2007).

There are many factors that determine the level of sports participation on an individual level such as age, gender, education and job which cannot be altered by politicians. But the latter can and have the responsibility to modify those factors relating to sports supply, which may not be the same everywhere. What is available affects the choice of individuals. 


\section{Sociological and Psychological Factors}

Recent studies on sports participation have enquired new research areas developing new theories based on sociological and psychological theories. These efforts highlight other significant constraints in relation to an individual's behavior (Beaton et al., 2009; Henderson, 2009). Psychological theories examine the motivations behind behaviour (Beaton et al., 2009). Social theories investigate the connection between sport and physical activity and the construction of an individual's identity (Bourdieu, 1997).

The psychological and sociological frameworks incorporate different approaches to accentuate how several factors and constraints external to the individual influence behaviour (Lera-López and Rapún-Gárate, 2011). Three different approaches are the preventive medicine approach which highlights the part played by health motivations; the self-determination theory and the theory of participation which identifies one of the four stages of motivation: awareness, attraction, attachment and allegiance related to an individual participation.

The social network of an individual is instrumental for sport participation. Becker (1974) had referred to an individual's shared characteristics with peers as being part of a component of a commodity whereby it produces a sort of joint consumption of sport. If the network includes mostly active individuals than it is more likely, the individual is more likely to increase personal utility. As a matter of fact, peers' characteristics have also the potential to discourage sport participation if no value is placed on it. In a recent study, Hallmann and Breuer (2014) claim that social recognition influences sport participation and sport frequency, though in different ways. They concluded that friends significantly impact one's decision to take part in sport, while the involvement of parents in sport affects sport frequency in a positive and significant way.

From a sociological standpoint, education is an important player in explaining sports participation. Lera-López and Rapún-Gárate (2011) argue that based on empirical evidence by Breuer and Wicker (2008), Downward (2007) and Humphreys and Ruseski (2007), higher education levels and ease of access to inexpensive facilities imply increased awareness of sport benefits and positive habits on the part of individuals.

From a psychological viewpoint, different studies have examined the motivations behind individuals' decisions to take up sports in the context that over a lifespan their tastes and preferences are constantly evolving. Several groups of motivational influences, such as health and fitness, enjoyment and recreation, relaxation, appearance, socialization and competition/challenge, have been identified by Fridberg (2010) and Garcia and Llopis (2011). Empirical evidence has emphasized the relevance of motives such as recreation, fitness, competition and professional development to explain sports participation (Lera-López and Rapún-Gárate, 2011). 
In another study, Davey et al. (2009) applied the "Self-Determination Theory" framework to categorize various extrinsic or intrinsic motivations into five groups, namely appearance, competition/challenge, enjoyment, health and fitness and social.

In a qualitative study investigating the determinants of sports participation among recently retired people, Sport England (2006) identified physical benefits, weight control, independence and social, mental and emotional benefits as the main internal motivators. External motivators included media and families emphasizing the benefits of sports participation. There were no big differences in motivators between genders.

Lim et al. (2011) explains that different factors motivate the participation of youths and adults in sport. Physical competence, skill development, enjoyment, challenge and social acceptance motivate young people to continue their involvement in sport. In contrast, barriers or reasons for drop outs in youths include amongst others, lack of playing time, lack of fun, expense, coach conflicts, travel and adult support. In contrast, motivations, experiences and constraints related to sport participation of adults vary over the course of a life time. Changes in age and choice of activity and differences in gender affect the motivations of adults. Constraints vary from lack of time in mid-life to less physical ability in later life.

A study on adolescent swimmers' sport participation aimed to understand their training patterns and the roles of parents, coaches, peers and siblings concluded that the inevitable requirement of suitably structured programmes and the instability of adolescent athletes' relationships with others (Fraser-Thomas et al., 2008). Digging deeper into the study, some new elements emerge. Sport programmes should not simply focus on the development of the performing athlete but also emphasize the physical and psychosocial development. The philosophy behind such programmes should be communicated to coaches, athletes and parents. Sport participation is affected by the quality of the coaches, their supporting and caring behaviour in addition to excellent communication skills and technical expertise. Dropouts spoke, of coach favoritism and less attention to weaker swimmers. Parents keep an open communication with their children while their expectations should not be too high or inflexible. A supportive group of peers and siblings is vital to the continued involvement in sport during adolescence.

\section{Country Level Factors, Government Support and Lifestyle Factors}

An international comparative analysis by Ruseski and Maresova (2014) refers to country level factors in the form of "aggregate economic performance, policies directly related to sport, and policies indirectly related to sport" that have an influence on participation in sports and physical activity. This supports the importance of government policies in influencing individual's decision to be physically active which should be based on institutional factors and sport-related 
policies. An important claim in this study, states that broad-based participation is not developed through the provision of resources directed towards elite athletes.

A positive association was obtained between the quality of government and public health expenditure with sport participation (Van Tuyckom, 2011a). Downward et al. (2014) have investigated both government variables such as real GDP and expenditure and lifestyle factors such as smoking and drinking. Increase in participation and frequency levels resulted from government expenditures in sport. The study also revealed a negative relationship of smoking for both males and females and a decline in sport participation and frequency linked to the consumption of alcohol which could potentially be the result of a substitute effect in leisure activities particularly for males.

An individual's lifestyle also needs to be considered in relation to sports participation. This could disclose important information about his or her inclinations. Eberth and Smith (2010) found that smoking had a negative impact on duration and frequency of sport participation in both men and women. Alcohol delivered an unclear result, most probably due to the social aspect of sport, team sports. Another possibility could be that a compensation effect takes place whereby a healthy behaviour makes up for an unhealthy one.

Wheeler (2011) explored the significance of family sporting cultures with some noteworthy outcomes. Parents employed a clear set of goals, practices and strategies to encourage and support their children's participation in sports. Parents' background, whether sporting or non- had an impact on their goals vis-à-vis their children's participation. They transported their children to training and games, provided positive feedback and watched them performing.

\section{The Determinants of Sport Participation in General}

Most of the studies have mainly investigated the determinants of general sports participation. The levels of physical activity of 10 to 18 years Portuguese adolescents were analyzed by Seabra et al. (2007). Age, gender, mother and sibling physical activity, peer influence and socioeconomic status were the main demographic and socio-cultural correlates that were significantly associated with physical activity. Perhaps unexpectedly, physical education teachers were found as having no influence.

Downward and Riordan (2007) explored the determinants of both the decision to take part in sport and the frequency of participation in the United Kingdom. Findings were consistent with other analysis and indicated the important role of social and personal capital. Berger et al. (2008) examined sport participation of Canadian adolescents aged between 15 to 19 years. Gender, self-perceptions, household and community contexts and competing behaviours were identified as the main drivers. 
In their study, Hallman et al. (2011) identified differences relating to the influence of micro and macro level factors on sports participation in medium-sized and metropolitan municipalities. Some of their findings contrast with some of the literature. Young people in medium-sized municipalities with a high level of human capital, no migration background and caring for children and relatives for longer time periods were more likely to be physically active. In metropolis, people were more likely to practice sport if they had a high weekly workload. Differences at the macro level include a larger supply of facilities in medium-sized municipalities. Swimming pools play a significant role on sports participation in the metropolis while sport fields are more essential in medium-sized municipalities.

Time constraints and family structure were the two economic factors examined in more detail by Ruseski et al. (2011). The study was carried out among residents of Rheinberg, Germany using a unique primary data source and focused on the decisions to participate and for how long. Results are essentially consistent with findings in prior research. The decisions made by individuals to take part in sports and the time spent participating are influenced by the presence of children in the household and time spent caring for children and relatives.

\section{The Determinants of Participation in Different Sports}

In a study which investigated data from the United Kingdom General Household Survey held in 1996, Downward (2004) found that males' participation was strongly associated with cycling, football, rugby, running, squash and weight training while females were more oriented towards participation in keep fit, horse riding, netball and swimming. In general, participation increased when children were absent from households except for football, netball and swimming.

Leslie et al. (2004) determined that while on one hand younger women prefer aerobics, basketball, cycling and netball, older ones' favor cycling and swimming. Men are more active in cycling, jogging and swimming. In a study examining 40 different sports and recreational activities, Lera-López and Rapún-Gárate (2011) identified several motivational factors including competitivness, professional, recreational, slimness and fitness in addition to age and number of sporting activities as positive determinants which enhance the frequency of sports participation. In contrast, several employment and occupational status categories such as selfemployed, manager and unemployed are key negative determinants.

Humphreys and Ruseski (2007) investigated participation in physical activity in the United States by means of a nationally representative sample collected between 1998 and 2000. Walking was the most common form of exercise. Running and working out at home or club was also very popular while outdoor recreation and group sports are comparatively low. A heterogeneous relationship was experienced between participation and various factors like gender, education, marital status and education. 
Ifedi (2008) studied the level and type of Canadian sports participation in 2005. The study was based on the General Social Survey and 19,597 Canadians were interviewed through a Computer Assisted Telephone Interviewing (CATI). Results revealed that all age groups in Canada experienced a decrease in sports participation. It established that women's favorite participating sports are football, golf, skiing, swimming and volleyball while men are more active in baseball, basketball, football, golf and ice hockey. This study confirmed previous trends in this field of research. Men were more active than women. Sports participation increases with higher educational background and income level. Native Canadians were more likely to be active than immigrants.

By means of the demographic-economic model, which had been applied earlier by Breuer and Wicker (2008) to investigate general sport participation, Breuer et al. (2011) depicted the profiles of German sports participants in different sports. They were able to predict participation across ten different sports in Germany. Each sport was influenced by different variables of the demographic-income model. Individual and non-organized sport such as cycling, running and swimming were the most popular activities. The most frequently practiced team sport was football which was positively influenced by being male, young, less-educated and of foreign nationality. Being male, well-educated, native and high income made the typical profile for tennis. Being female and medium aged positively influenced participation in walking/hiking while being male, medium-aged, well-educated and native of the country were key characteristics of swimming participants. Breuer et al., (2011) suggest the integration of motivational questions for participation and environmental factors in the form of sports supply and effects of infrastructure into a multi-level analysis.

\section{The Determinants of Sports Participation at EU Level}

Recent years have experienced increasing literature investigating the determinants of sports participation at EU level. Traditionally, international comparisons between European countries on sport participation were based on preconceived notions such as geographical, historical and political determinants. The first study to offer empirically stratification of countries using data from the 2004 Eurobarometer survey about 25 European member states was Van Tuyckom (2011b). Although the 2004 Eurobarometer survey has several limitations, the author was able to group countries into 'six sporting worlds' each representing European differences in sports participation. Malta was placed in cluster three named 'average non-organized sporting countries' together with Cyprus and Slovenia. This study suggested a differentiated approach in policy strategies aimed to raise sport participation levels in Europe, since the intensity and provision of sport in the six worlds are rather diverse.

Using the same data to analyze gender and age differences in regular sports participation, Van Tuyckom et al. (2010) reported that men were more likely to be 
active "in Belgium, France, Greece, Latvia, Lithuania, Slovakia, Spain, and the UK," while the same holds true for women in "Denmark, Finland, Sweden, and the Netherlands". Scandinavian countries are more active than countries in the Mediterranean Sea area, while West Europeans score better than East Europeans. Findings show variable results when examining the association between gender and sport participation based on different age categories.

For the first time, Downward et al. (2014) investigated a more complete set of correlates of sports participation at European Level using data from the Eurobarometer survey of 2009 by focusing not only on the let's say typical factors such as economic, socio-demographic and lifestyle, but also analyzing the availability of sports infrastructure, motivational factors and government support. Findings suggest that government support, club membership and certain motivations have the potential to improve participation. The provision of sports facilities is more relevant for males who also play sport primarily to integrate socially in contrast to females who aim to improve self-esteem. Females have accession problems to sport arising from constraints related to current facility provision, household and time obligations and travelling issues. Such gender differences propose a distinctive approach in government policies to enhance sports participation.

\section{Sports Participation in Norway}

"Norway boasts particularly high levels of sports participation as well as sports club membership and young Norwegians are the quintessential sporting omnivores" (Green et al., 2015). Sports policy-makers across various European countries and non- have regularly observed the success of Scandinavian and other Nordic countries in achieving higher levels of sport participation in terms of forms, contexts, rates and frequencies. Their aim was to identify potential initiatives they can employ in their own country.

Green et al. (2015) explain that there were more Norwegians playing more sport more often. A sport and physical recreation culture is deeply rooted in the Norwegian society and is supported by favorable socio-economic circumstances, high standard of living, equality between genders, abundant sporting facilities, a school system that keenly promotes physical activity, a strong voluntary sports clubs sector and high levels of parental contribution. The authors have accredited the huge cultural power of sport in Norway which has registered amongst other positive results, considerable increases in participation among young people especially females, a growth in lifestyle sports and more lifelong participants. They also emphasize that the greater socio-economic conditions in Norway as well as in other Scandinavian countries to be 'unrealistic benchmarks' for the development of sports.

\section{Conclusion}


This paper has focused on the main determinants of sports participation by individuals. With this review of existing literature, we give an insight into what drives people to participate or not in sports and physical activities. A number of models have been developed to study this phenomenon, including the "IncomeLeisure Trade-Off Model of Labour Supply" which uses the household as its unit of analysis and "The SLOTH framework" which investigates the economic decisions influencing participation in physical activity. One can observe that sports participation is gender specific whereby men are more active than women in physical activity. One of the reasons for this is time constraint, in that women are the primary care providers for children and family members. Another determinant of sport participation is age, whereby sports participation decreases as age increases.

However, walking is seen as being the activity of choice with increase in age. Spending capacity and education also have an impact on sports activity. Men spend more money than women on sports and individuals with a higher education and better income tend to spend more money than people with a lower educational background.

Whilst socio-economic factors determine the level of activity, one must not underestimate the effect of infrastructure. Studies show that physical activity is positively correlated with the availability of suitable sport infrastructure. People tend to be more active if they have the facilities available to them and men and women are inclined to use different amenities when these are available. Whilst gender, age, education and infrastructure influence the rates of physical activity, nowadays researchers have understood that they need to look at the sociological and psychological factors, which encourage sports participation as well. An individual's social network will have a big impact on the level of sports activities he/she will engage in. Moreover, research has shown that friends as well as parents significantly impact one's decision to participate in sports activities.

\section{References:}

Anokye, N.K., Pokhrel, S. and Fox-Rushby, J. 2014. Economic analysis of participation in physical activity in England: implications for health polic. International Journal of Behavioral Nutrition and Physical Activity, 11, https://doi.org/10.1186/s12966-0140117-9.

Balbinotto, G., Godoy, M.R. and Massuquetti, A. 2012. Determinants of Female Participation in Sports Activities: Evidence from Brazil. International Journal of Gynecology and Obstetrics, 119S3, S531-S867.

Beaton, A.A., Funk, D.C. and Alexandris, K. 2009. Operationalizing a Theory of Participation in Physically Active Leisure. Journal of Leisure Research, 41(2), 177203.

Becker, G. 1965. A Theory of the Allocation of Time. Economic Journal, 75, 493-513.

Becker, G. 1974. A theory of social interactions. Journal of Political Economy, 82(6), 1063 1093.

Berger, I.E., O’Reilly, N., Parent, M.M., Seguin, B. and Hernandez, T. 2008. Determinants 
of sport participation among Canadian adolescents. Sport Management Review, 11(3), 277-307.

Bourdieu, P. 1997. The Forms of Capital. In A.H. Halsley, H. Launder, P. Brown and A. Start Wells (Eds.), Education, culture, economy and society. Oxford: Oxford University Press.

Breuer, C. 2006. Sportpartizipation in Deutschland - ein demo-okonomisches Modell. Sportwissenschaft, 36(3), 292-305.

Breuer, C., Hallmann, K. and Wicker, P. and Feiler, S. 2010. Socio-economic patterns of sport demand and ageing. European Review of Aging and Physical Activity, 7(2), 61 70.

Breuer, C., Hallmann, K. and Wicker, P.2011. Determinants of sport participation in different sports. Managing Leisure, 16(4), 269-286.

Breuer, C. and Wicker, P. 2008. Demographic and economic factors concerning the conclusion in the German sport system: A micro-analysis of the year 1985 to 2005. European Journal for Sport and Society, 5(1), 33-42.

Breuer, C. and Wicker, P. 2009. Sports clubs in Germany. In C. Breuer (Ed.), Sport Development Report 2007/2008. Analysis of the sports clubs' situation in Germany, Abbreviated Version. Cologne: Sportverlag Strauss.

Chad, K.E., Reeder, B.A., Harrison, E.L., Ashworth, N.L., Shephard, S.M., Schultz, S.A., Bruner, G., Fisher, K.L. and Lawson, J.A. 2005. Profile of physical activity levels in community-dwelling older adults. Medicine and Science in Sports and Exercise, 37(10), 1774-1784.

Cawley, J. 2004. An Economic Framework for Understanding Physical Activity and Eating Behaviors. American Journal of Preventive Medicine, 27, 117-125.

Davey, J., Fitzpatrick, M., Garland, R. and Kilgour, M. 2009. Adult participation motives: Empirical evidence for a workplace exercise programme. European Sport Management Quarterly, 9, 141-162.

Dixon, M. 2009. From their perspective: A qualitative examination of physical activity and sport programming for working mothers. Sport Management Review, 12, 34-48.

Downward, P. 2004. On Leisure Demand: A Post Keynesian Critique of Neoclassical Theory. Journal of Post Keynesian Economics, 26(3), 371-394.

Downward, P. 2007. Exploring the economic choice to participate in sport results from the 2002 general household survey. The International Review of Applied Economics, 21(5), 633-653.

Downward, P., Dawson, A., Dejonghe, T. 2009. Sports Economics. Theory, Evidence and Policy. Elsevier, Amsterdam.

Downward, P., Lera-Lopez, F., Rasciute, S. 2011. The Zero-Inflated ordered probit approach to modelling sports participation. Economic Modelling, 28(6), 2469-2477.

Downward, P., Lera-López, F., Rasciute, S. 2014. The correlates of sports participation in Europe. European Journal of Sport Science, 14(6), 592-602.

Downward, P., Rasciute, S. 2010. The relative demands for sports and leisure in England. European Sport Management Quarterly, 10, 189-214.

Downward, P., Rasciute, S. 2011. Does sport make you happy? An analysis of the wellbeing derived from sports participation. International Review of Applied Economics, 25, 331-348.

Downward, P., Rasciute, S. 2015. Exploring the covariates of sport participation for health: an analysis of males and females in England. Journal of Sports Sciences, 33(1), 67-76.

Downward, P. and Riordan, J. 2007. Social interactions and the demand for sport: An economic analysis. Contemporary Economic Policy, 25(4), 518-537. 
Eberth, B., Smith, M.D. 2010. Modelling the participation decision and duration of sporting activity in Scotland. Economic Modelling, 27, 822-834.

Eime, R.M., Casey, M.M., Harvey, J.T., Sawyer, N.A., Symons, C.M., Payne, W.R. 2015. Socioecological factors potentially associated with participation in physical activity and sport: A longitudinal study of adolescent girls. Journal of Science and Medicine in Sport, 18, 684-690.

Eime, R.M., Harvey, J.T., Sawyer, N.A., Craike, M.., Symons, C.M., Polman, R.C.J., Payne, W.R. 2013. Understanding the Contexts of Adolescent Female Participation in Sport and Physical Activity. Research Quarterly for Exercise and Sport, 84(2), 157-166.

Federico, B., Falese, L., Marandola, D., Capelli, G. 2013. Socioeconomic differences in sport and physical activity among Italian adults. Journal of Sports Sciences, 31, 451-458.

Fraser-Thomas, Cote, J., Deakin, J. 2008. Understanding dropout and prolonged engagement in adolescent competitive sport. Psychology of Sport and Exercise, 9, 645-662.

Fridberg, T. 2010. Sport and exercise in Denmark, Scandinavia and Europe. Sport in Society, $13,583-592$.

Garcia, J., Lera-Lopez, F., Suarez, M.J. 2011. Estimation of a structural model of the determinants of the time spent on physical activity and sport: Evidence for Spain. Journal of Sports Economics, 12, 515-537.

García, M., Llopis, R. 2011. Survey about sport habits in Spain 2010. Madrid, CIS and CSD.

Gratton, C., Taylor, P. 2000. Economics of Sport and Recreation. Taylor \& Francis, London, Spon Press.

Green, B.C. 2005. Building sport programs to optimize athlete recruitment, retention, and transition: Toward a normative theory of sport development. Journal of Sport Management, 19, 233-253.

Green, G., Thurston, M., Vaage, O., Roberts, K. 2015. We're on the right track, baby, we were born this way'! Exploring sports participation in Norway. Sport, Education and Society, 20(3), 285-303.

Hallmann, K., Breuer, C. 2014. The influence of socio-demographic indicators economic determinants and social recognition on sport participation in Germany. European Journal of Sport Science, 14(sup1), S324-S331.

Hallmann, K., Wicker, P., Breuer, C., Schönherr, L. 2011. Interdependency of sport supply and sport demand in German metropolitan and medium-sized municipalities - findings from multi-level analyses. European Journal for Sport and Society, 8 (1/2), 65-84.

Hallmann, K., Wicker, P., Breuer, C., Schönherr, L. 2012. Understanding the importance of sport infrastructure for participation in different sports - findings from multi-level modelling. European Sport Management Quarterly, 12(5), 525-544.

Haug, E., Torsheim, T., Sallis, J.F., Samdal, O. 2008. The characteristics of the outdoor school environment associated with physical activity. Health Education Research, 25(2), 248-256.

Henderson, K.A. 2009. A paradox of sport management and physical activity interventions. Sport Management Review, 12, 57-65.

Henderson, K.A., Hickerson, B.D. 2007. Women and leisure: Premises and performances uncovered in an integrative review. Journal of Leisure Research, 39, 591-610.

Hinrichs, T., Trampisch, U., Burghaus, I., Endres, H., Klaaßen-Mielke, R., Moschny, A., Platen, P. 2010. Correlates of sport participation among community-dwelling elderly in Germany: a cross-sectional study. European Review of Aging and Physical Activity, $7(2), 105-115$.

Hovemann, G., Wicker, P. 2009. Determinants of sport participation in the European Union. European Journal for Sport and Society, 6(1), 49-57. 
Humphreys, B., Ruseski, J. 2006. Economic determinants of participation in physical activity and sport. Working Paper Series of the International Association of Sports Economics (IASE), 06-13.

Humphreys, B.R., Ruseski, J.E. 2007. Participation in physical activity and government spending on parks and recreation. Contemporary Economic Policy, 25(4), 538-552.

Humphreys, B.R., Ruseski, J.E. 2009. The economics of participation and time spent in physical activity, Working Paper 2009-09. Department of Economics, Institute for Public Economics, University of Alberta, Edmonton.

Ifedi, F. 2008. Sport Participation in Canada, 2005. Ottawa, Statistics Canada: Culture, Tourism and the Centre for Education Statistics.

Karusisi, N., Thomas, F., Meline, J., Chaix, B. 2013. Spatial accessibility to specific sport facilities and corresponding sport practice: The Record Study. International Journal of Behavioral Nutrition and Physical Activity, 10(48).

Klein, T. 2009. Determinanten der Sportaktivität und der Sportart im Lebenslauf. Kölner Zeitschrift für Soziologie und Sozialpsychologie, 61(1), 1-32.

Lera-López, F., Rapún-Gárate, M. 2011. Determinants of sports participation and attendance: differences and similarities. International Journal of Sports Marketing \& Sponsorship, 12(2), 167-190.

Leslie, E., Cerin, E., Gore, C.J., Bauman, A., Owen, N. 2004. Gender, age and educationalattainment differences in Australian adults' participation in vigorous sporting and fitness activities. Journal of Physical Activity and Health, 1(4), 377-388.

Lim, S.Y., Warner, S., Dixon, M., Berg, B., Kim, C., Newhouse-Bailey, M. 2011. Sport Participation Across National Contexts: A Multilevel Investigation of Individual and Systemic Influences on Adult Sport Participation. European Sport Management Quarterly, 11(3), 197-224.

Limstrand, T., Rehrer, N.J. 2008. Young people's use of sports facilities: A Norwegian study on physical activity. Scandinavian Journal of Public Health, 36, 452-459.

Liu, Y.D., Taylor, P., Shibli, S. 2009. Sport equity: benchmarking the performance of English public sport facilities. European Sport Management Quarterly, 9(1), 3-21.

Lubans, D.R., Sylva, K., Morgan, P.J. 2007. Factors associated with physical activity in a sample of British secondary school students. Australian Journal of Educational and Developmental Psychology, 7, 22-30.

Prins, R.G., Mohnen, S.M., Van Lenthe, F.J., Brug, J., Oenema, A. 2012. Are neighbourhood social capital and availability of sports facilities related to sports participation among Dutch adolescents? International Journal of Behavioral Nutrition and Physical Activity, 9(90).

Reimers, A.K., Wagner, M., Alvanides, S., Steinmayr, A., Reiner, M. 2014. Proximity to Sports Facilities and Sports Participation for Adolescents in Germany. PLoS ONE, 9(3), e93059.

Robertson, J., Emerson, E. 2010. Participation in sports by people with intellectual disabilities in England: A brief report. Journal of Applied Research in Intellectual Disabilities, 23(6), 616-622.

Ruseski, J.E., Humphreys, B.R., Hallmann, K., Breuer, C. 2011. The interplay of family structure, time constraints, and sport participation. European Review of Aging and Physical Activity, 8(2), 57-66.

Ruseski, J.E., Maresova, K. 2014. Economic Freedom. Sport Policy and Individual Participation in Physical Activity: An international Comparison. Contemporary Economic Policy, 32(1), 42-55.

Scheerder, J., Vos, S. 2011. Social stratification in adults' sports participation from a time- 
trend perspective. Results from a 40-year household study. European Journal for Sport and Society, 8, 31-44.

Scheerder, J., Thomis, M., Vanreusel, B., Lefevre, J., Renson, R., Vanden Eynde, B., Beunen, G.P. 2006. Sports participation among females from adolescence to adulthood. A longitudinal study. International Review for the Sociology of Sport, 41(3/4), 413-430.

Seabra, A.F., Mendonca, D.M., Thomis, M.A., Malina, R.M., Maia, J.A. 2007. Sports participation among Portuguese youth 10 to 18 years. Journal of Physical Activity and Health, 4(4), 370-380.

Slater, A., Tiggemann, M. 2010. Uncool to do sport: A focus group study of adolescent girls' reasons for withdrawing from physical activity. Psychology of Sport and Exercise, 11, 619-626.

Sport England. 2006. Understanding participation in sport: what determines sports participation among recently retired people? Sport England, London.

Sport England. 2010. Active people survey 4. London, Sport England. Available from: http://www.sportengland.org/research/active_people_survey/active_people_survey_4.a spx. .

Snape, R., Binks, P. 2008. Re-thinking sport: Physical activity and healthy living in British South Asian Muslim communities. Managing Leisure, 13(1), 23-35.

Stahl, T., Ritten, A., Nutbeam, D., Bauman, A., Kannas, L., Abel, T. 2001. The importance of the social environment for physically active lifestyle - results from an international study. Social Science \& Medicine, 52, 1-10.

Stamatakis, E., Chaudhury, M. 2008. Temporal trends in adults' sports participation patterns in England between 1997 and 2006: The Health Survey for England. British Journal of Sports Medicine, 42(11), 901-908.

Stamm, H., Lamprecht, M. 2011. Swiss sports participation in an international perspective. European Journal for Sport and Society, 8(1), 15-29.

Taks, M., Renson, R., Vanreusel, B. 1994. Of sport, time and money: An economic approach to sport participation. International Review for the Sociology of Sport, 29(4), 381-395.

Taks, M., Scheerder, J. 2006. Youth sports participation styles and market segmentation profiles: Evidence and applications. European Sport Management Quarterly, 6(2), 85121.

Van Bottenburg, M. 2005. Sport participation in the EU: Trends and differences. Available from: http://www.mulierinstituut.nl.

Van Tuyckom, C. 2011a. Macro-environmental factors associated with leisure-time physical activity: A cross-national analysis of EU countries. Scandinavian Journal of Public Health, 39, 419-426.

Van Tuyckom, C. 2011b. Six sporting worlds. A cluster analysis of sports participation in the EU-25. Quality \& Quantity, 47(1), 441-453.

Van Tuyckom, C., Scheerder, J. 2008. Sport for all? Social stratification of recreational sport activities in the EU-27. Kinesiologia Slovenica, 14(2), 54-63.

Van Tuyckom, C., Scheerder, J. 2010a. Sport for all? Insight into stratification and compensation mechanisms of sporting activity in the 27 European Union member states. Sport, Education and Society, 15, 495-512.

Van Tuyckom, C., Scheerder, J. 2010b. A multi-level analysis of social stratification patterns of leisure-time physical activity among Europeans. Science and Sports, 25(6), 304311.

Van Tuyckom, C., Scheerder, J., Bracke, P. 2010. Gender and age inequalities in regular sports participation: A cross-national study of 25 European countries. Journal of Sports 
Sciences, 28, 1077-1084.

Walseth, K. 2008. Bridging and bonding social capital in sport-experiences of young women with an immigrant background. Sport, Education and Society, 13(1), 1-17.

Wheeler, S. 2011. The Significance of Family Culture for Sports Participation. Sociology of Sport, 47(2), 235-252.

Wicker, P., Breuer, C., Pawlowski, T. 2009. Promoting sport for all to age specific target groups - the impact of sport infrastructure. European Sport Management Quarterly, 9(2), 103-118.

Wicker, P., Hallmann, K., Breuer, C. 2012. Micro and Macro level determinants of sport participation. Sport, Business and Management: An International Journal, 2(1), 51-68.

Xiong, H. 2007. The evolution of urban society and social changes in sports participation at the grassroots in China. International Review for the Sociology of Sport, 42(4), 441471.

Zimmermann-Sloutskis, D., Wanner, M., Zimmermann, E., Martin, B. 2010. Physical activity levels and determinants of change in young adults: A longitudinal panel study. International Journal of Behavioral Nutrition and Physical Activity, 7. 